\title{
Response of the Hodgkin-Huxley Neuron to a Periodic Sequence of Biphasic Pulses
}

\author{
L.S. BORKOWSKI \\ Department of Physics, Adam Mickiewicz University, Umultowska 85, 61-614 Poznań, Poland
}

(Received May 27, 2013; in final form October 20, 2013)

\begin{abstract}
Electric stimulation of various parts of the nervous system is a widely used therapeutic method and a principle of operation of prosthetic devices. Its usefulness has been proven in areas such as treatment of neurological disorders and cochlear prostheses. However the dynamic mechanisms underlying these applications are not well understood. In order to shed some light on this problem we study the response of the Hodgkin-Huxley neuron subject to periodic train of biphasic rectangular current pulses. One of the simpler ways to understand the behavior of such a nonlinear system is the analysis of the global bifurcation diagram in the period-amplitude plane. For short pulses the topology of this diagram is approximately invariant with respect to the pulse polarity and shape details. The lowest excitation threshold for charge-balanced input was obtained for cathodic-first pulses with an inter-phase gap approximately equal to $5 \mathrm{~ms}$. The firing rate of the Hodgkin-Huxley neuron stimulated at the frequency of its natural resonance is a square root function of the pulse amplitude. At nonresonant frequencies the quiescent state and the firing state coexist and transition to firing is a discontinuous one. We found a multimodal transition in the regime of irregular firing between the 2:1 and 3:1 locked-in states. This transition separates the regime of odd-only multiples of the stimulus period from the regime where modes of both parities participate in the response. A strong antiresonant effect was found between the states $3: 1$ and $4: 1$, where the modes $(2+3 n): 1$, where $n=0,1,2, \ldots$, were entirely absent. The antiresonant effects at high stimulation frequency, such as multimodal transition, may provide an explanation for the therapeutic mechanism of deep brain stimulation.
\end{abstract}

DOI: $10.12693 /$ APhysPolA.125.145

PACS: 87.19.11, 87.19.ln

\section{Introduction}

Stimulation of neural fibers with a train of electric current pulses has become standard procedure in several areas of clinical practice. This encompasses, but is not limited to, cochlear and retinal prostheses [1-3], deep brain stimulation (DBS) $[4,5]$, as well as high-frequency conduction block of peripheral nerves [6]. Typically such devices should minimize charge and energy per pulse. The minimum current amplitude required for the neuron to spike and its dependence on stimulation frequency and pulse shape are among the basic properties measured in experiment. The injected charge should be kept at safe levels, mainly to avoid changes in the chemical content of tissue near the electrode which could damage tissue $[7,8]$ or cause the electrode corrosion [9]. Usually the injected charge needed by the neuron to spike is minimized by the shortest possible width whereas optimization of the pulse energy occurs at certain larger width [10-14]. Selection of the most efficient waveform shape and duration, such that delivered charge, energy and power are simultaneously optimized is a difficult task $[15,16]$. Most studies indicate that non-rectangular pulses are more energy efficient than rectangular ones $[11,13,14,16]$.

The general stimulation wave form in DBS applications consists of a brief cathodic pulse, followed by a delay, and then a charge-balancing anodic pulse [17]. The change of the wave form affects stimulation thresholds of local cells and fibers of passage differently, thus aiding in selecting the stimulation target. Both symmetric and asymmetric biphasic charge-balanced wave forms in se- lective stimulation of the CNS were investigated [5, 18]. Also most cochlear implants use charge-balanced biphasic (BP) stimulation. Studies of auditory nerve fibers (ANF) found that threshold can be reduced [19, 20] by introducing a delay between standard BP pulses commonly used in cochlear implants.

Some of the DBS research is focused on the dynamics of the cortico-basal-ganglia-thalamo-cortical network. It is thought that optimal response to DBS occurs at resonant frequencies of the network [21-23]. However, DBS is less effective at lower frequencies and the therapeutic effect is most beneficial above $100 \mathrm{~Hz}$, well above the frequency range normally considered for basal ganglia processing. Since there is some evidence of $300 \mathrm{~Hz}$ subthalamic oscillations [24] it has been suggested that multiple natural periods may be involved in parkinsonism due to multiple network loops.

However, there is another possible mechanism for disruption of network activity patterns. Recent studies of resonant neurons revealed the presence of multimodal transition (MMT), occurring at approximately $2.5 f_{\text {res }}$, where $f_{\text {res }}$ is the natural resonance frequency, where the parity of response modes is changed [25-28]. This effect has an antiresonant character. It involves both a chaotic behavior and a significant slowing down of average response frequencies in the vicinity of MMT. It would therefore be useful to investigate if this phenomenon is sensitive to details of the stimulus wave form and whether it exists also for charge-balanced inputs.

Studies of electrically stimulated ANFs show that at low stimulation rates these fibers fire regularly, in-step 
to applied stimulus, whereas at high stimulation rates the response is highly irregular. Irregular firing and desynchronization among a group of ANFs is usually attributed to irregular synaptic input and physiological noise $[29,30]$. It must be kept in mind however that neurons themselves are strongly nonlinear systems and are capable of irregular firing even in the absence of noise $[25,26]$. O'Gorman et al. showed recently [31, 32] that a dynamic instability is a plausible explanation of firing irregularities in stimulated ANF. Using the FitzHughNagumo (FHN) model [33], they obtained a positive Lyapunov exponent which is consistent with experiments, where mutual desynchronization between similarly stimulated fibers was observed. Their study is consistent with our analysis of the MMT within the $\mathrm{HH}$ [25-27] and Morris-Lecar [28] models. Since multimodal dynamics is common in electrically stimulated ANFs [34-40], the current article may help to understand the response of ANFs to stimulation by a train of high-frequency current pulses.

The aim of this work is not the search for the most optimal charge and energy-efficient pulse shape. The focus is on (i) proving the invariance of the global bifurcation diagram of a resonant neuron with respect to pulse shape, polarity, and inter-phase gap (IPG), (ii) finding the optimal IPG for a rectangular biphasic wave form, and (iii) proving that the dynamic instability in the form of the recently discovered multimodal transition exists also for biphasic pulses, which makes this phenomenon interesting in the context of clinical applications.

\section{Methods}

We analyzed the response of the $\mathrm{HH}$ neuron to periodic biphasic charge balanced pulses. The requirement of charge neutrality was imposed to avoid tissue damage. Such stimuli were investigated experimentally [20, 41, 42] and theoretically by several groups [19, 20, 43, 44].

We considered the model with the classic parameter set and rate constants [45],

$$
C \frac{\mathrm{d} V}{\mathrm{~d} t}=-I_{\mathrm{Na}}-I_{\mathrm{K}}-I_{\mathrm{L}}+I_{\mathrm{app}},
$$

where $I_{\mathrm{Na}}, I_{\mathrm{K}}, I_{\mathrm{L}}, I_{\mathrm{app}}$, are the sodium, potassium, leak, and external current, respectively. $C=1 \mu \mathrm{F} / \mathrm{cm}^{2}$ is the membrane capacitance. The input current was a periodic set of rectangular steps of height $I_{0}$ and width $\tau$. The calculations were carried out with the time step of $0.001 \mathrm{~ms}$ and were run for $40 \mathrm{~s}$, discarding the initial $4 \mathrm{~s}$. The differential equations were integrated using the fourth-order Runge-Kutta scheme implemented by the author. None of the publicly available programs were used.

Figure 1 shows the form of biphasic pulses used in the calculation. The emphasis of this work is on the charge-balanced stimulus shown in Fig. 1a. We investigated how the amplitude, period $T_{i}$, width $\tau$, and delay $\tau_{1}$ between the onsets of the two phases, influence the neuron's response. Stimuli of this form are used in cochlear $[19,20,43,44]$ and visual $[1,3]$ neural prostheses, as well

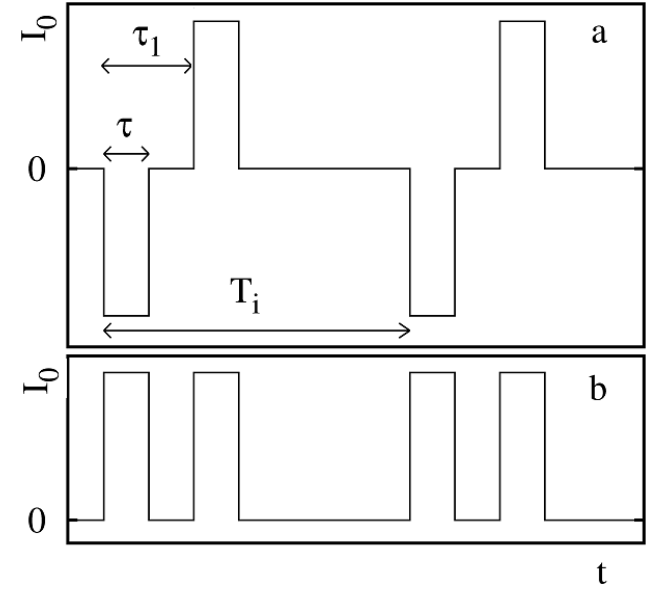

Fig. 1. Stimulus wave form used in the calculations: (a) charge-balanced biphasic form with delay, and (b) monophasic pulses with delay. The delay between the onset of the cathodic and anodic phase is $\tau_{1}$ and $\tau$ is the width of each phase.

as deep brain stimulation [13] and muscle stimulation [9]. Electrical stimulation protocols have been used to learn about cortical function [46] and vestibulo-ocular reflex eye movements in chinchillas [47]. High-frequency biphasic current pulses may also be useful in reversible peripheral nerve block that would be desirable in some clinical applications [6]. We also considered monopolar biphasic pulses, shown in Fig. 1b, and calculated threshold dependence on IPG at different stimulation frequencies.

\section{Results}

Figure 2 shows the global bifurcation diagram in the period-amplitude plane for a charge-balanced biphasic current with no delay between the cathodic and anodic part, i.e. $\tau=\tau_{1}=0.6 \mathrm{~ms}$. The numbers in the figure show the location of various states locked-in to the stimulus. For example 2 denotes the state $2: 1$, where there is one action potential for each two stimulus pulses. The notation $p$ : $q$ means $q$ output spikes for every $p$ current pulses. The type of the near-threshold response depends strongly on stimulation frequency. The firing rate $f_{0} / f_{i}$ is a continuous function of the current amplitude near the resonance [27], $T_{i} \sim T_{\text {res }} \approx 17 \mathrm{~ms}$, where $T_{\text {res }}$ is the neuron's preferred interspike interval (ISI).

Resonant stimulation periods are sometimes defined as the location of the membrane voltage maxima [48, 49]. However this definition makes $T_{\text {res }}$ depend on pulse magnitude $I_{0}$. In the context of this article we prefer to use a related but slightly different description. The resonances can be identified by the minima of the excitation threshold. Both definitions are equivalent at the threshold. In Fig. 2 they are located at $T_{i} \approx 17 \mathrm{~ms}$ and $34 \mathrm{~ms}$. The local threshold maxima are associated with antiresonances. In Fig. 2 they occur at $T_{i} \approx 25 \mathrm{~ms}$ and just below $6 \mathrm{~ms}$. It is clear from Fig. 2 and from earlier analysis $[27,28]$ 


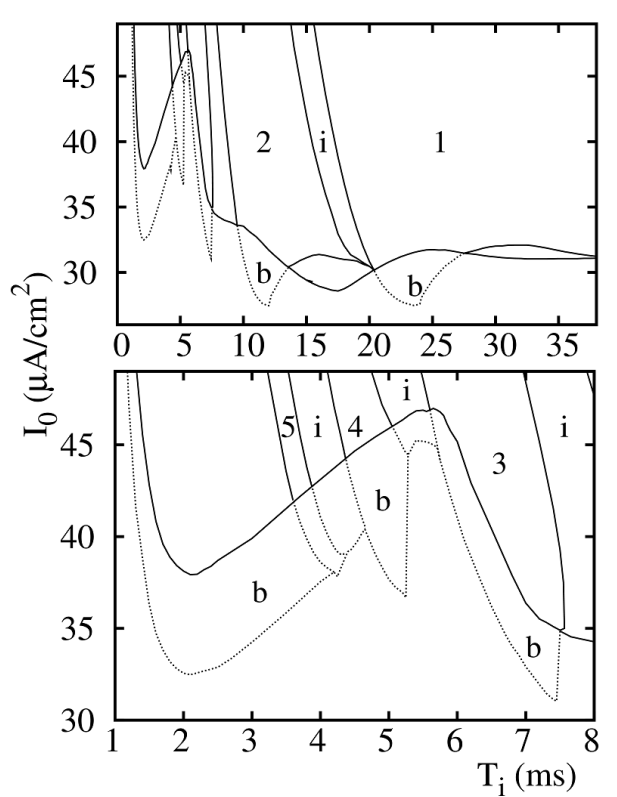

Fig. 2. Top part: response diagram for a biphasic stimulus with no delay between the anodic and the cathodic part of a pulse, $\tau=\tau_{1}=0.6 \mathrm{~ms}$. The high frequency limit of the same diagram is shown in more detail in the bottom part. The numbers $1,2, \ldots$ indicate the zones of 1:1, 2:1, and higher locked-in states; symbols $i$ and $b$ are used to denote regions of irregular and bistable behavior, respectively. Borders of bistable regions are marked with a dotted line. In the zones between the solid and dotted lines the firing state coexists with the silent one. The resonance near $T_{i}=17 \mathrm{~ms}$ and $I_{0}=30 \mu \mathrm{A} / \mathrm{cm}^{2}$, where the minimum of the excitation threshold occurs, is dominated by the $3: 1$ state and higher order states. Its mode-locking structure is essentially identical to that of monophasic monopolar stimulation shown in Fig. 3 of Ref. [27]. The firing rate $f_{0} / f_{i}$, where $f_{0}$ and $f_{i}$ are the average response and stimulation frequency, continuously decreases to 0 at the tip of the resonance, $f_{0} / f_{i} \sim\left(I_{0}-I_{\mathrm{th}}\right)^{1 / 2}$. The second resonance near $T_{i}=34 \mathrm{~ms}$ is occupied by states of order higher than 1:1 (see also Ref. [27]). Also at this resonance the firing rate depends continuously on $I_{0}$. Bottom part: the regime of high stimulation frequency in more detail. There is a strong antiresonance near $T_{i}=5.5 \mathrm{~ms}$.

that antiresonances are accompanied by bistable behavior. Most of the regime of high stimulation frequency has antiresonant character.

In bistable regions the neuron can be in one of two states. One is a firing state, such as 1:1, 2:1, 3:1, etc. and the other is the quiescent state. Boundaries of these areas were found using a continuation algorithm, in which end values from previous iteration were used as initial conditions for a new parameter set.

Periodically stimulated neural oscillators are known to phase-lock with the stimulus. Examples of such locking are shown in Fig. 3. The 1:1 locking is displayed in Fig. 3a, where there is an action potential for every

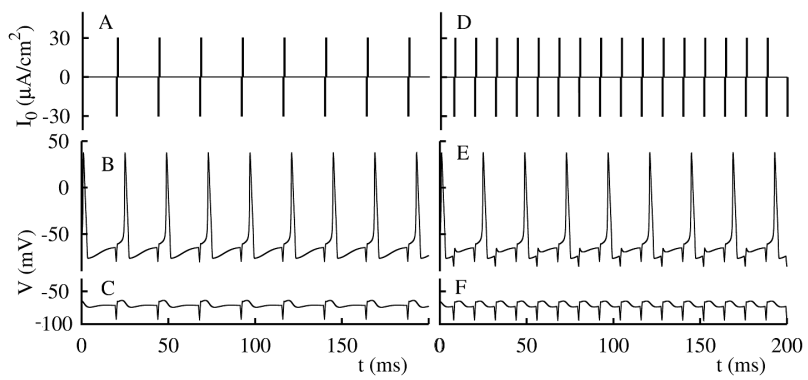

Fig. 3. Examples of solutions in bistable zones for stimulus with no inter-phase gap, $\tau=\tau_{1}=0.6 \mathrm{~ms}$, and amplitude $I_{0}=30 \mu \mathrm{A} / \mathrm{cm}^{2}$, and stimulus period (A) $T_{i}=24 \mathrm{~ms}$, (D) $T_{i}=12 \mathrm{~ms}$. (B) Firing solution locked 1:1 in response to stimulus shown in (A). (C) Non-firing solution in response to stimulus (A). (E) Firing solution locked $2: 1$ in response to stimulus shown in (D). (F) Non-firing solution in response to stimulus (D).

stimulus pulse. Mode-locking of order $n: 1$, where $n$ is an integer greater than 1 , can be obtained for higher stimulation frequencies or just above threshold for $T_{i} \approx T_{\text {res }}[27]$. Figure 3 shows an example of $2: 1$ locking for $T_{i}=12 \mathrm{~ms}$. Both regular and irregular patterns, corresponding to noninteger ratios of the average output period $T_{0}$ to the input period $T_{i}$, also exist.

The overall topology of this diagram closely resembles the result for monophasic monopolar pulses [27]. In the high frequency limit the threshold is a nonmonotonic function of $T_{i}$ with a local maximum near $T_{i}=5.5 \mathrm{~ms}$, which is approximately $1 / 3$ of the resonance period $T_{\text {res }}$. In other words, the $\mathrm{HH}$ neuron is least likely to respond when driven with the frequency $3 f_{\text {res }}$. Below $T_{i}=8 \mathrm{~ms}$ the entire perithreshold region is bistable. Here the quiescent state coexists with either a locked-in or a chaotically firing state. Which of these solutions is obtained depends sensitively on initial conditions. It is worth noting that nonmonotonic behavior of the hearing threshold was observed in experiments in cochlear implant users [50]. However more detailed experimental studies of threshold dependence on stimulation frequency are needed before comparisons could be made. Special attention must be paid to the possibility of bistable behavior at the threshold.

The firing frequency depends nonmonotonically on $T_{i}$. Figure 4 shows $f_{0}$ vs. $T_{i}$ for $I_{0}$ slightly above threshold. The largest deviation from the resonant frequency occurs between the $2: 1$ and $3: 1$ states, near $T_{i}=7.5 \mathrm{~ms}$. This is a signature of a dynamic instability associated with the competition of even and odd modes [25]. Sample $V(t)$ runs very close to this instability and is shown in Fig. 5. The odd multiples of the stimulus period clearly dominate. Out of 18 ISIs visible in Fig. 5 there are only two even multiples of $T_{i}$ in Fig. 5 .

In an earlier article we showed that the $\mathrm{HH}$ neuron responding to a periodic sequence of monophasic pulses undergoes a multimodal odd-all [25] and even-all tran- 


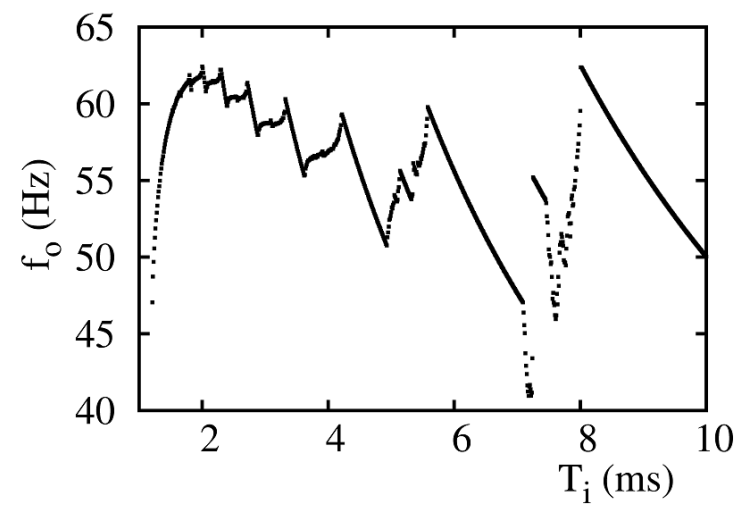

Fig. 4. The firing frequency in the limit of high stimulation frequency for a biphasic pulse at $I_{0}=47 \mu \mathrm{A} / \mathrm{cm}^{2}$, with no inter-phase delay, $\tau=\tau_{1}=0.6 \mathrm{~ms}$. The response between the mode-locked intervals is nonmonotonic and highly irregular.

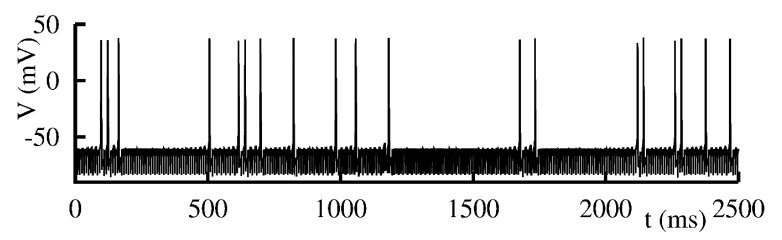

Fig. 5. Sample $V(t)$ runs for $T_{i}=8.35 \mathrm{~ms}$, very close to the multimodal transition. Here $\tau=\tau_{1}=0.6 \mathrm{~ms}$, and the stimulus amplitude is $I_{0}=35 \mu \mathrm{A} / \mathrm{cm}^{2}$. The shown ISI sequence is $3,5,41,13,3,7,15,19,9,15$, $59,7,46,3,14,3,11,11$, in units of $T_{i}$. The ISIs are mostly odd multiples of $T_{i}$.

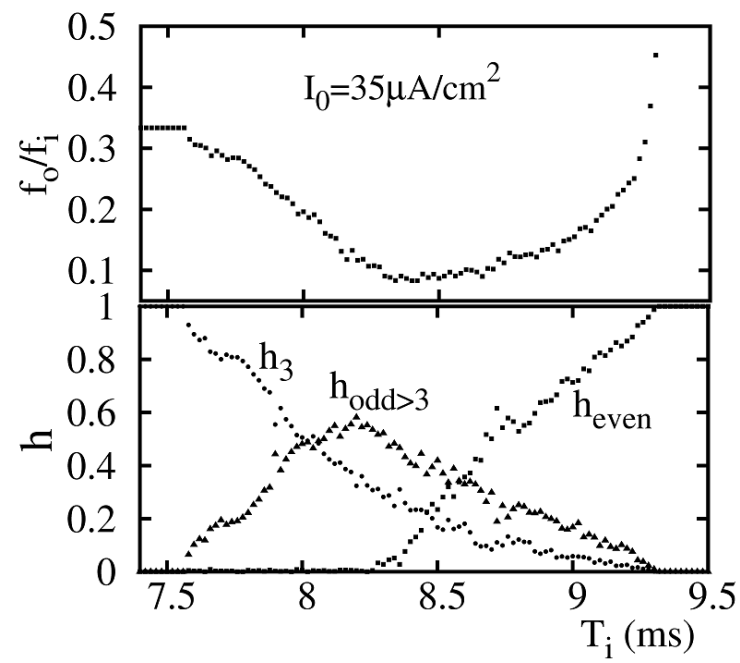

Fig. 6. The firing rate (top) and the histogram weight of odd and even modes (bottom) in the vicinity of the odd-all multimodal transition, $T_{i} \approx T_{\mathrm{mm}}$, for stimulation with a sequence of biphasic pulses of amplitude $I_{0}=35 \mu \mathrm{A} / \mathrm{cm}^{2}$. Even modes appear only above the minimum of $f_{0} / f_{i}$. An interspike interval is classified as belonging to the $n$-th mode if it falls between $(n-1 / 2) T_{i}$ and $(n+1 / 2) T_{i}$. sition [27] in some regions of parameter space. Figure 6 shows the minimum of the firing rate occurring between the 2:1 and 3:1 locked states for pulse amplitude $I_{0}=35 \mu \mathrm{A} / \mathrm{cm}^{2}$. The weight of even modes is nonzero only for $T_{i}$ above the minimum of $f_{0} / f_{i}$.

For $T_{i}$ below $T_{\mathrm{mm}}$ only odd modes exist. The role of the mode parity is easier to understand if we remember that $2 T_{i}$, where $T_{i} \approx 8.5 \mathrm{~ms}$ is approximately equal to the resonant period $T_{\text {res }}$. The competition of odd and even modes in Fig. 6 accompanies the transition between the resonant and antiresonant regime. The vanishing of even modes below $T_{i} \approx 8.4 \mathrm{~ms}$ and a significant decrease of the firing rate are a clear signature of entering the antiresonant regime.

The dependence of the firing rate on pulse amplitude $I_{0}$ in the vicinity of the threshold is qualitatively different on both sides of the multimodal transition, see Fig. 7 . For $T_{i}<T_{\mathrm{mm}}$ the firing rate depends discontinuously on pulse amplitude. However the size of the discontinuity decreases as $T_{i}$ approaches $T_{\mathrm{mm}}$. For $T_{i} \geq T_{\mathrm{mm}}, f_{0}$ is a continuous, nearly linear function of $I_{0}$. In this regime the average firing rate may be arbitrarily small.

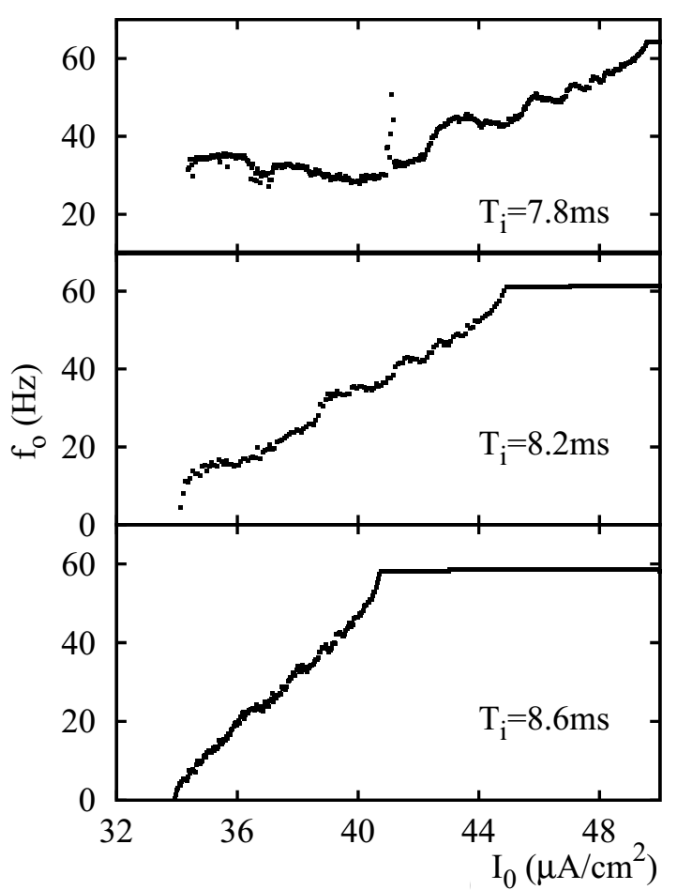

Fig. 7. The firing rate as a function of the stimulus amplitude $I_{0}$ at fixed $T_{i}$ near the multimodal transition.

The transition occurs at $T_{\mathrm{mm}} \approx 8.4 \mathrm{~ms}$. Here $\tau=\tau_{1}=$ $0.6 \mathrm{~ms}$.

At high frequencies, for $T_{i}<5.5 \mathrm{~ms}$, the bistable zones encompass not only phase-locked states with integer ratios of $T_{0} / T_{i}$, but also states with no regular response pattern. Dependence of the firing rate on the current pulse amplitude in one of such zones is shown in Fig. 8. Here the irregularly firing state coexists with a quiescent state. The firing rate depends discontinuously on stimulus amplitude in this regime. 


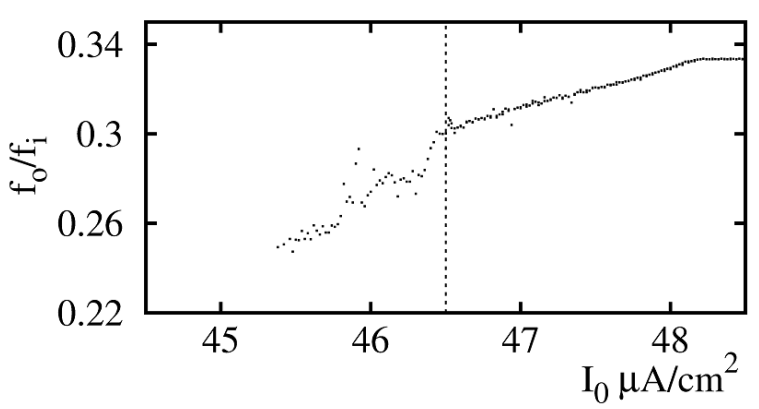

Fig. 8. The firing rate as a function of the stimulus amplitude for $T_{i}=5.5 \mathrm{~ms}$ and no delay between the phases of the pulse, $\tau=\tau_{1}=0.6 \mathrm{~ms}$. The vertical broken line separates bistable and monostable regions.

Another interesting effect occurs between the 3:1 and 4:1 states at high frequencies. Bands of $T_{0}$ vs. $T_{i}$ values are shown in Fig. 9. Note the absence of all modes of the form $(5+3 n): 1$, where $n$ is a nonnegative integer. This is a consequence of the fact that the product $(5+3 n) T_{i}$ falls in an antiresonant regime for $T_{i} \approx 5.5 \mathrm{~ms}$. A typical $V(t)$ dependence in this situation is shown in Fig. 10. This is a qualitative effect, independent of a particular pulse shape.
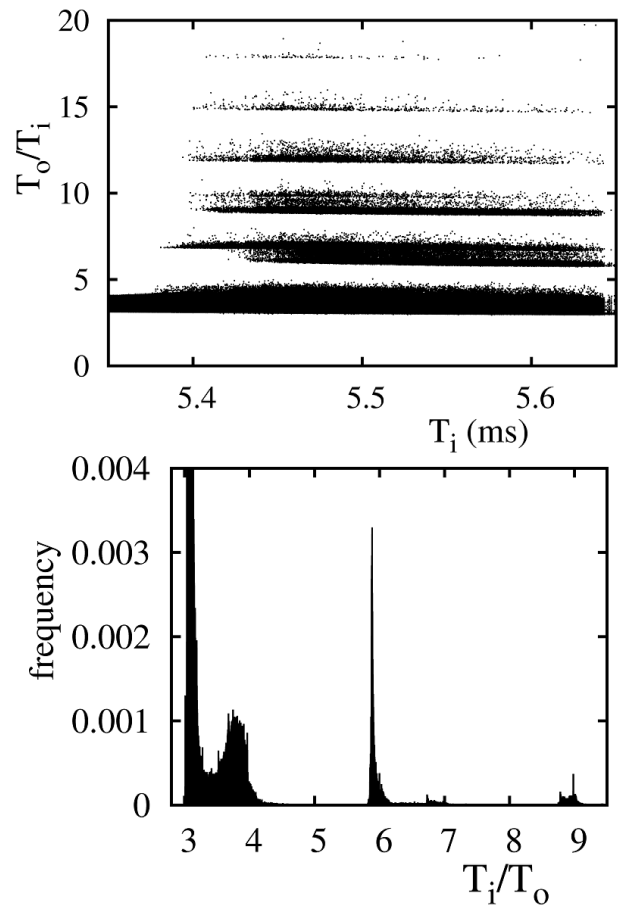

Fig. 9. Top: the ratio of the output period $T_{0}$ to the input period $T_{i}$ between the $3: 1$ and $4: 1$ entrained states for a biphasic pulse at $I_{0}=45.8 \mu \mathrm{A} / \mathrm{cm}^{2}$. Bottom: histogram of response modes at $T_{i}=5.55 \mathrm{~ms}$ and $I_{0}=45.8 \mu \mathrm{A} / \mathrm{cm}^{2}$. The calculations were carried out for stimulus with no inter-phase gap, $\tau=\tau_{1}=0.6 \mathrm{~ms}$. The histogram bin size is $0.01 \mathrm{~ms}$. Note the absence of modes $5,8,11,14$, and 17 .

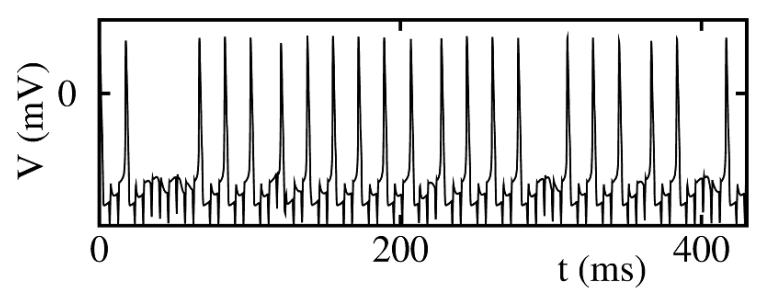

Fig. 10. Sample $V(t)$ dependence for $T_{i}=5.55 \mathrm{~ms}$. Here $\tau=\tau_{1}=0.6 \mathrm{~ms}$, and the stimulus amplitude $I_{0}=$ $45.8 \mu \mathrm{A} / \mathrm{cm}^{2}$ is slightly above threshold. The modes $3: 1$ and $4: 1$ dominate, with no clear separation between them. The modes $(5+3 n): 1$, where $n=0,1,2, \ldots$, do not appear in the neuron's response.

Figure 11 shows the excitation threshold as a function of the delay between the anodic and the cathodic phases of the pulse, for four choices of the stimulus period. In each case the optimum $\tau_{1}$ is about $5 \mathrm{~ms}$. If the input period is in the antiresonant regime, i.e. for $T_{i}=11$ and $23 \mathrm{~ms}$, there are two thresholds because of bistability, both of which have minima at similar values of $\tau_{1}$. The upper threshold where the transition to firing occurs through a subcritical Hopf bifurcation, has a maximum at approximately $\tau_{1}=13 \mathrm{~ms}$. This antiresonant feature occurs at the same $\tau_{1}$ also in stimulation by pulses separated by $T_{i}=34 \mathrm{~ms}$. Lower thresholds for biphasic cathodic-first stimuli were also observed in Refs. [51-53] and calculated by Smit et al. [44] in an ANF model containing persistent sodium and slow potassium currents.

The minimum threshold is obtained for the stimulus frequency tuned to the natural resonance of the neuron. The resonance period sets also an upper limit of IPG, for which the interaction between the cathodic and anodic phase is noticeable, see bottom part of Fig. 11. The spike-triggered average of stimulus $I(t)$ for isolated spikes in the $\mathrm{HH}$ model driven by Gaussian random noise current with a short correlation time also indicated the preference of the neuron for approximately $5 \mathrm{~ms}$ separation between the negative and positive parts of the current [54]. This is a reflection of the $\mathrm{HH}$ neuron internal dynamics in which the sodium channel remains open for about $5 \mathrm{~ms}$ from the stimulus onset. The bifurcation diagram with an optimal IPG, $\tau_{1} \approx 5 \mathrm{~ms}$, shown in Fig. 12 closely resembles Fig. 2. The only significant difference is the shift of the boundary between the quiescent state and firing states to lower values of $I_{0}$.

The precise location of the tip of main resonance $T_{\text {res }}$ depends on the size of IPG. For fixed $\tau$ the resonance period grows with increasing $\tau_{1}$, see Fig. 13. This slowing down is due to the cathodic pulse which delays the firing cycle. At the optimal IPG, $\tau_{1}=5 \mathrm{~ms}$, the tip of the resonance is shifted by approximately $1 \mathrm{~ms}$ relative to its position for monophasic excitatory pulses.

The firing rate at the main resonance frequency, shown in Fig. 14, is a square root function of the pulse amplitude $f_{0} \sim\left(I_{0}-I_{\mathrm{th}}\right)^{1 / 2}$, where $I_{\mathrm{th}}$ is an approximate 


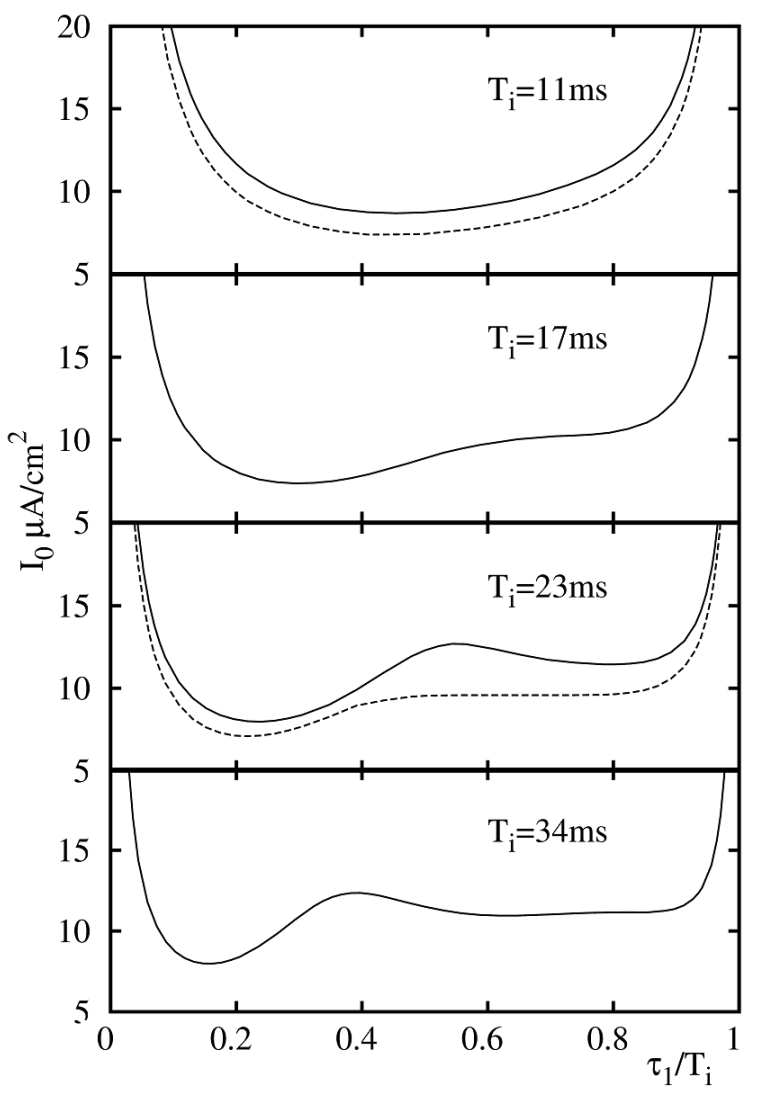

Fig. 11. Excitation edge for different interpulse separation as a function of time difference between the cathodic and anodic phase. From top to bottom: $T_{i}=$ $11,17,23$, and $34 \mathrm{~ms}$, for stimulation by rectangular current pulses of width $\tau=0.6 \mathrm{~ms}$ and height $I_{0}$. For different pulse frequencies the minimum occurs at $\tau_{1} \approx 5 \mathrm{~ms}$. The threshold is bistable for $T_{i}=11 \mathrm{~ms}$ and $23 \mathrm{~ms}$, where both the firing solution and the quiescent state exist between the broken line and the solid line.

threshold. This implicates that the transition to firing occurs via a saddle node bifurcation. This is a property of the neuron's internal dynamics and is independent of a stimulus shape. However, it is important to realize that the character of the bifurcation at the resonance changes as the width of the pulse increases beyond optimal [27]. For pulse widths approaching the time scale of the resonance, the firing rate becomes a discontinuous function of $I_{0}$. The transition to firing occurs then via a subcritical Hopf bifurcation and there is a bistable zone near the threshold at all frequencies.

An illustration of slow firing rates near the resonance is shown in Fig. 15. It is quite surprising that this aspect of the $\mathrm{HH}$ neuron dynamics was not known until recently [27]. We can expect similar slowing down in many other models of resonant neurons as well, although the exact details of this behavior will depend on the relative magnitude of time scales of each model.

The threshold for biphasic monopolar pulses is shown in Fig. 16. For $T_{i} \leq T_{\text {res }}$ the most regular input signal with equally spaced pulses, $\tau_{1}=T_{i} / 2$, is also the least

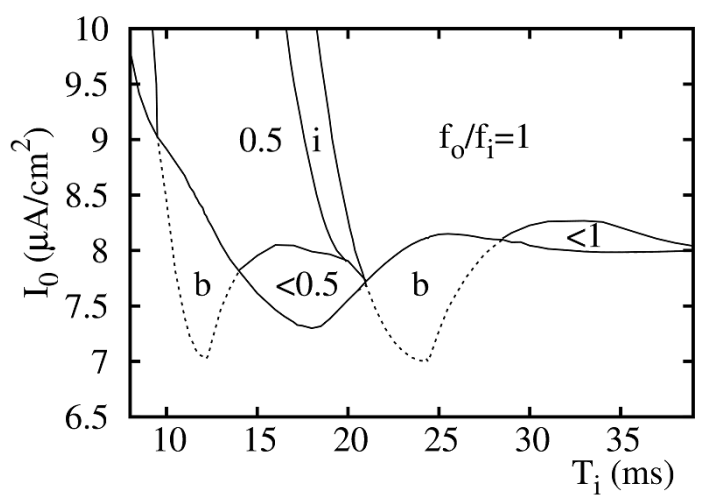

Fig. 12. Response diagram for stimulus with an optimal inter-phase gap, $\tau_{1}=5 \mathrm{~ms}, \tau=0.6 \mathrm{~ms}$. Symbols $i$ and $b$ indicate a zone of irregular firing and bistability, respectively. Boundaries of bistable zones are marked with a dotted line. The resonance area near $T_{i}=17 \mathrm{~ms}$ is dominated by the $3: 1$ state and higher order states. In this zone the firing rate is below 0.5 and decreases almost continously to 0 at the tip of the resonance. The second resonance near $T_{i}=34 \mathrm{~ms}$ is occupied by modes higher than 1:1. In this regime the firing rate is below 1 .

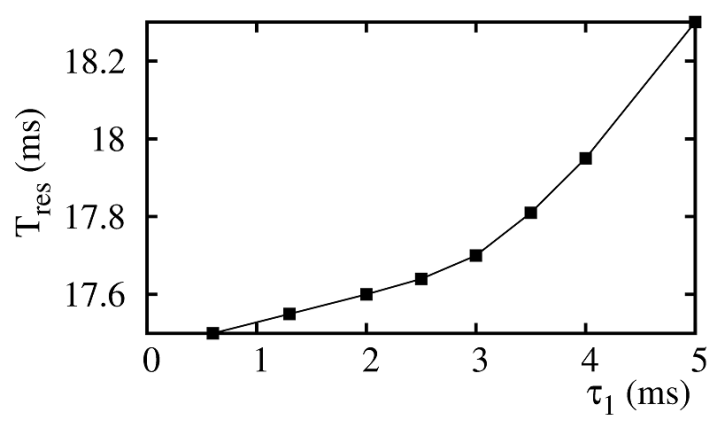

Fig. 13. Dependence of the location of the resonance tip on the inter-phase gap $\tau_{1}$. The phase width is fixed at $\tau=0.6 \mathrm{~ms}$

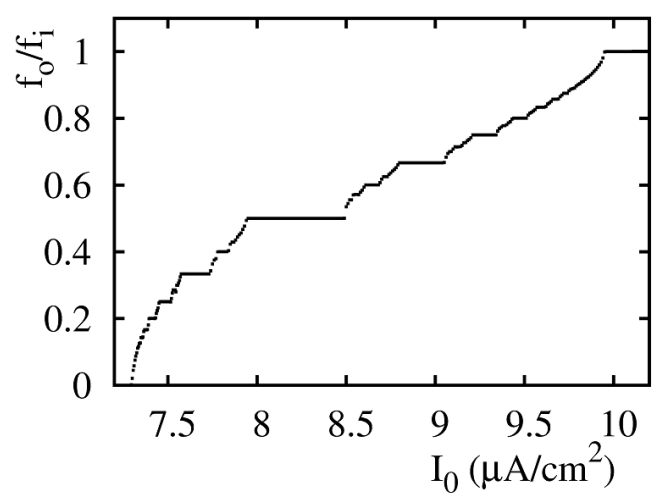

Fig. 14. Firing rate as a function of the stimulus amplitude in the resonant regime, $T_{i}=18.3 \mathrm{~ms}$. Here $\tau=0.6 \mathrm{~ms}, \tau_{1}=5 \mathrm{~ms}$. Note the continuous dependence of the firing rate on $I_{0}, f_{0} \sim\left(I_{0}-I_{\mathrm{th}}\right)^{1 / 2}$, near the threshold. 


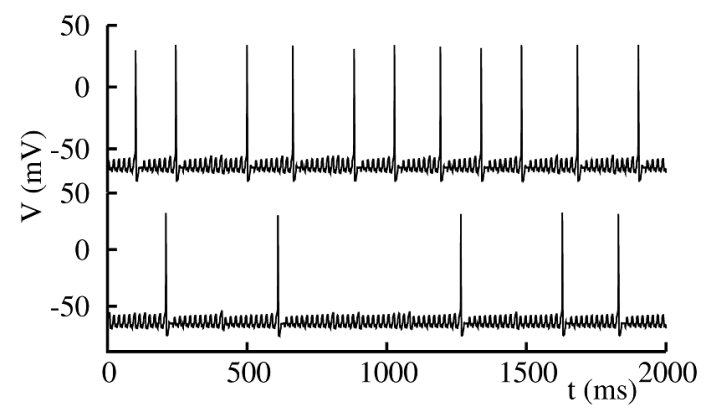

Fig. 15. Sample $V(t)$ dependence near the resonance at $T_{i}=18.2 \mathrm{~ms}$, for stimulation by biphasic pulses with $\tau=0.6 \mathrm{~ms}, \tau_{1}=5 \mathrm{~ms}$. The current amplitude in the top and bottom part is $I_{0}=7.33 \mu \mathrm{A} / \mathrm{cm}^{2}$, and $7.303 \mu \mathrm{A} / \mathrm{cm}^{2}$, respectively.

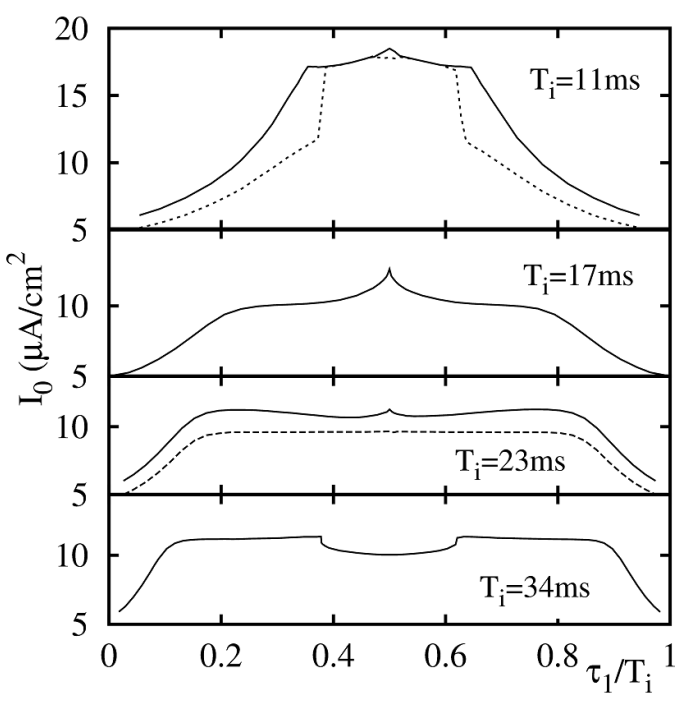

Fig. 16. Excitation edge for a double pulse as a function of separation between the leading and the trailing phase. The inter-phase gap is scaled in units of the stimulation period $T_{i}$. The diagrams from top to bottom are drawn for $T_{i}=11,17,23$, and $34 \mathrm{~ms}$, respectively.

likely to elicit spiking. The notation here is the same as in Fig. 1. Any deviation from the perfect regularity of the stimulus for $\tau_{1}=T_{i} / 2$ and $T_{i} \leq T_{\text {res }}$, either by means of a deterministic or stochastic perturbation leads to a decrease of threshold.

Comparing Fig. 12 to Fig. 2, we can conclude that the topology of the response diagram does not depend on the size of IPG for $\tau_{1}<5 \mathrm{~ms}$. The same is likely to be true for all types of periodic short pulses irrespective of details of their time dependence. This is an illustration of the well known fact that the $\mathrm{HH}$ neuron has a charge threshold property. When a relatively strong current is delivered in a short time, the voltage change is mainly determined by the capacitive current [55-57].

In neural electrical stimulation the main safety factors are the amount of charge transferred in a single pulse, $Q_{\mathrm{th}}$, and energy per spike, $E_{\mathrm{th}}$, needed to evoke spiking,
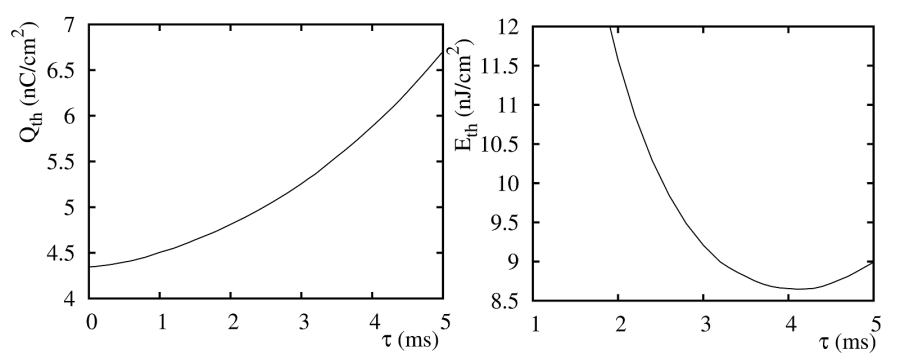

Fig. 17. Threshold charge (left part) and threshold energy (right part) as a function of $\tau$ for a resonant stimulation, $T_{i}=17 \mathrm{~ms}$, by a biphasic periodic sequence of pulses with an optimal inter-phase gap, $\tau_{1}=5 \mathrm{~ms}$. The minimum charge occurs at $\tau \rightarrow 0$ which means that the most effective pulses are those with the shortest possible width. The pulse energy has a minimum near $\tau=4 \mathrm{~ms}$.

$$
\begin{aligned}
& Q_{\mathrm{th}} \sim \int_{0}^{\tau} I_{\mathrm{th}}(t) \mathrm{d} t, \\
& E_{\mathrm{th}} \sim \int_{0}^{\tau} I_{\mathrm{th}}^{2}(t) \mathrm{d} t,
\end{aligned}
$$

where $I_{\mathrm{th}}$ is the threshold current amplitude. Figure 17 shows the threshold charge at $T_{i}=17 \mathrm{~ms}$ as a function of the phase width $\tau$ for $\tau_{1}=5 \mathrm{~ms}$. The charge-duration curve in Fig. 17 implies the use of short pulses in stimulation protocols if the injected charge is to be minimized. In practice, pulses lasting tens of microseconds approach the minimum charge condition sufficiently well and are often a reasonable solution in the design of neural prostheses. During this relatively short time one may be able to avoid Faradaic reactions that would occur at higher levels of total charge with longer pulses. Similar conclusion was reached in a study of Sahin and Tie [11] who investigated effects of non-rectangular wave forms on threshold both experimentally and theoretically within a simple local model of a mammalian nerve $[58,59]$. Their model is similar to the $\mathrm{HH}$ model except for the absence of the potassium channel. However if the main limiting factor is the energy delivered per pulse, then finite $\tau$ is preferable, see Fig. 17. Qualitatively similar result was obtained for different wave forms in Ref. [11].

\section{Discussion}

The global bifurcation diagram of the $\mathrm{HH}$ neuron stimulated by biphasic charge-balanced pulses is very similar to the diagram obtained in the study of monophasic stimulation [27]. Charge-balancing has no effect on topology of the global bifurcation diagram in the period-amplitude plane. Figure 2 closely resembles results obtained for the monophasic stimulation [27]. These properties are quite general, provided the width of the current pulses is sufficiently small, which implies $\tau_{1}<5 \mathrm{~ms}$ for the classic $\mathrm{HH}$ parameter set, and should hold for other, non-rectangular pulse shapes.

Studies of threshold behavior of resonant neurons may help to design future cochlear implants. The excitation 
threshold is a very sensitive function of IPG. When the anodic part of the pulse follows immediately the cathodic part the magnitude of threshold current pulses at the main resonant frequency is almost $I_{0} \approx 30 \mu \mathrm{A} / \mathrm{cm}^{2}$ (see Figs. 11 and 2) for the phase width of $\tau=0.6 \mathrm{~ms}$. When the IPG is increased, the threshold decreases, reaching $I_{0} \approx 7.3 \mu \mathrm{A} / \mathrm{cm}^{2}$ (see Fig. 12) for the near-optimal inter-phase separation of $\tau_{1}=5 \mathrm{~ms}$. In the monophasic stimulation by current pulses of the same phase width the threshold at $T_{\text {res }}$ is approximately $I_{0} \approx 10 \mu \mathrm{A} / \mathrm{cm}^{2}$ [27]. It is interesting to note that in the monophasic case the perfectly periodic stimulus has a higher threshold than a signal with unequal intervals between subsequent pulses, see Fig. 16. Results in Figs. 11 indicate that IPG giving the lowest firing threshold of the $\mathrm{HH}$ membrane is approximately $4-5 \mathrm{~ms}$. The IPG required to minimize threshold is approximately the same as the IPG which minimizes the energy delivered per pulse. The decrease of threshold associated with the increase of IPG is consistent with experimental measurements [19]. Similar result was obtained for biphasic pulses with long IPG in a phenomenological model of electrically stimulated human ANF [43]. Our results are also consistent with the work of Carlyon et al. [20] who studied human behavioral thresholds for trains of biphasic pulses applied to a single channel of cochlear implants as a function of IPG. The experimental threshold decreased for IPGs up to several milliseconds when the phases of the pulse were of opposite polarity.

The firing threshold of the $\mathrm{HH}$ model is a nonmonotonic function of the stimulation period with the minimum at the main resonance. A local minimum exists also at very high stimulation rate. At the resonant stimulation period and its multiples the solution is always unique and the firing rate is a continuous square-root function of the pulse amplitude $I_{0}, f_{0} / f_{i} \approx 2\left(I_{0} / I_{\text {th }}-1\right)^{1 / 2}$, where $I_{\text {th }}$ is the threshold. Thus $f_{0}$ can be arbitrarily low. The simplest way to obtain desired interspike separation $T_{0}$ in a resonant neuron of the $\mathrm{HH}$ type is to stimulate it with current pulses of amplitude

$$
I_{0} \approx I_{\mathrm{th}}\left(1+\frac{T_{\mathrm{res}}^{2}}{2 T_{0}^{2}}\right) .
$$

Naturally, it should be kept in mind that a resonant neuron need not have the dynamics of the $\mathrm{HH}$ model [28] and the square root dependence of the firing rate on current amplitude is not always available.

The optimal stimulation in clinical applications may include Gaussian noise superposed on the input signal [60]. Small amounts of noise have been shown to improve detection of faint signals, improve temporal resolution, and regularize strongly irregular firing in regimes of dynamic instabilities. Noise eliminates bistability at nonresonant frequencies [27]. However noise also broadens the local minima of excitation threshold which is not desirable in tasks such as selective stimulation of nerve fibers.

The recently discovered odd-all multimodal transition $[25,26]$, related to the vanishing of even response modes and accompanied by a deep local minimum of the firing rate, occurs also for charge-balanced stimuli. It is located between the 2:1 and 3:1 locked-in states and is most pronounced near excitation threshold. This phenomenon is a manifestation of the internal neuron dynamics. It is not related to a functional form of the stimulus. The change of the current pulse wave form shifts the excitation threshold, but does not affect the topology of the global bifurcation diagram. Also the frequency of the multimodal transition is invariant to the changes of the pulse shape.

O'Gorman et al. proposed that firing irregularity of $\mathrm{ANF}$ at high stimulation rates is due to a dynamic instability $[31,32]$. It is characterized by positive values of the Lyapunov exponent and explains both the sensitivity to small changes of the stimulus and the lack of synchronization of response of different ANFs to external drive [31]. Although O'Gorman et al. analyzed the instability from a different viewpoint, its dynamical mechanism in the FHN model is the same as in the HH model. The MMT $[25,26]$ and a dynamic instability $[31,32]$ associated with it are key properties of many resonant neurons. MMT implies threshold bistability at nonresonant stimulation frequencies above the natural frequency of the neuron [28]. The converse is not always true. Bistability at the threshold does not always imply the existence of MMT. As our recent study showed, neuron dynamics may be divided into four classes [28]. Only one of them displays MMT. Thus it is important to correlate the threshold studies with the search for the signatures of MMT in the ISI histograms.

This phenomenon may be relevant also in explaining the desynchronizing effect of high frequency stimulation on networks of oscillating neurons. Llinas et al. [61] claimed that many neurological disorders are caused by pathological resonant interaction between cortical and sub-cortical structures. Subsequent studies of network dynamics in the Parkinson disease found enhanced neural synchrony in the beta band $(13-30 \mathrm{~Hz})$ [62]. In fact, McIntyre and Hahn [63] point out that the strongest therapeutic effect in DBS treatment is obtained at frequencies of order $100 \mathrm{~Hz}$ and higher. They also propose that the optimal DBS frequencies are resonant frequencies of the cortico-basal-ganglia-thalamo-cortical loop or loops within this network. In our work the dominant high-frequency effects are of anti-resonant character. When some neurons in the loop are forced to fire chaotically by high-frequency stimulation, they are prevented from participating in a coordinated activity of a neural ensemble at their natural frequencies. Thus high-frequency anti-resonant effects, such as the multimodal transition, may help to explain the therapeutic mechanism of DBS.

The model considered here assumes intracellular current injection. Since clinical neurostimulation devices deliver current in the extracellular space, let us briefly comment how the presence of tissue affects the problem. Most bioelectric field models assume a purely resistive tissue and ignore capacitive, inductive and wave propagation effects. This approximation may not always be 
appropriate for stimulation by high-frequency pulses. In a realistic calculation both electrode and bulk tissue capacitance should be included [64]. Bossetti et al. [65] studied the problem and concluded that wave propagation and inductive effects can usually be neglected. However the quasi-static approximation is valid only for a specific range of tissue dielectric properties. The analysis of Tracey and Williams [66] showed that attenuation due to frequency-independent capacitance increases firing thresholds and the dispersion caused by the frequency-dependent effects may have an opposite influence, leading to lower threshold currents. Foutz and McIntyre pointed out that optimal pulse width depends on electrode geometry [13]. In the case of extracellular stimulation we expect the global bifurcation diagram, Figs. 2 and 12 , to have the same overall form, although details such as precise location of the threshold would be affected. MMT would occur at the same frequencies since it is a reflection of the internal dynamics of the neuron.

\section{Summary of results}

We would like to conclude the article by stating its main results:

- Stimulation by periodic biphasic pulses gives the same global bifurcation diagram in the periodamplitude plane as stimulation by monophasic pulses.

- There is a multimodal transition between the mode-locked states $2: 1$ and $3: 1$. This is a line of critical points $T_{\mathrm{mm}}, I_{\mathrm{mm}}$ such that below the transition, where $T_{i}<T_{\mathrm{mm}}$, only odd response modes exist. Even modes appear for $T_{\mathrm{mm}}<T_{i}$.

- We discovered a new phenomenon: the vanishing of the modes $2,5,8, \ldots$ in the regime of irregular firing between the $3: 1$ and $4: 1$ states.

- The maximum threshold is obtained near $T_{i} \approx$ $5.5 \mathrm{~ms}$, which is of order $T_{\text {res }} / 3$, between the entrained states $3: 1$ and $4: 1$.

- The minimum threshold at the tip of the main resonance is obtained for the inter-phase gap of about $5 \mathrm{~ms}$. This value of IPG is also associated with the minimum energy needed to elicit the response.

- The location of the minimum threshold depends on the size of IPG. For biphasic pulses with $\tau=\tau_{1}=$ $0.6 \mathrm{~ms}$ the tip of the resonance is located at $T_{i} \approx$ $17.5 \mathrm{~ms}$. For increasing IPG the resonance moves to higher values, reaching $18.3 \mathrm{~ms}$ for $\tau_{1}=5 \mathrm{~ms}$.

- The firing frequency is a continuous, square root function of the pulse amplitude at the resonance.

- The resonant neuron of the HH type can be forced to fire with arbitrarily low frequency $f_{0}$ by delivering current pulses of amplitude $I_{0} \approx I_{\mathrm{th}}(1+$ $\left.f_{0}^{2} / 2 f_{\text {res }}^{2}\right)$.
- The antiresonant effects at high stimulation frequency, such as the multimodal transition, may help to explain the therapeutic mechanism of DBS.

\section{References}

[1] E.M. Schmidt, M.J. Bak, F.T. Hambrecht, C.V. Kufta, D.K. O'Rourke, P. Vallabhanath, Brain 119, 507 (1996).

[2] C.A. Miller, P.J. Abbas, J.T. Rubinstein, B.K. Robinson, A.J. Matsuoka, G. Woodworth, Hear. Res. 119, 142 (1998).

[3] M.S. Humayun, E. de Juan Jr., J.D. Weiland, G. Dagnelie, S. Katona, R. Greenberg, S. Suzuki, Vision Res. 39, 2569 (1999).

[4] A.L. Benabid, P. Pollak, C. Gervason, D. Hoffmann, D.M. Gao, M. Hommel, J.E. Perret, J. de Rougemont, Lancet 337, 403 (1991).

[5] C.C. McIntyre, W.M. Grill, Ann. Biomed. Eng. 28, 219 (2000).

[6] C. Tai, W.C. de Groat, J.R. Roppolo, IEEE Trans. Biomed. Eng. 52, 1323 (2005).

[7] T.G. Yuen, W.F. Agnew, L.A. Bullara, S. Jacques, D.B. McCreery, Neurosurgery 9, 292 (1981).

[8] D.B. McCreery, W.F. Agnew, T.G. Yuen, L. Bullara, IEEE Trans. Biomed. Eng. 37, 996 (1990).

[9] D.R. Merrill, M. Bikson, J.G.R. Jefferys, J. Neurosci. Meth. 141, 171 (2005).

[10] J.L. Wessale, L.A. Geddes, G.M. Ayers, K.S. Foster, Ann. Biomed. Eng. 20, 237 (1992).

[11] M. Sahin, Y. Tie, J. Neural Eng. 4, 227 (2007).

[12] P. Danzl, A. Nabi, J. Moehlis, Discrete Contin. Dynam. Systems 28, 1413 (2010).

[13] T.J. Foutz, C.C. McIntyre, J. Neural Eng. 7, 066008 (2010) and references therein.

[14] S. Jezernik, T. Sinkjaer, M. Morari, J. Neural Eng. 7, 046004 (2010).

[15] A. Wongsarnpigoon, J.P. Woock, W.M. Grill, IEEE Trans. Neural Syst. Rehabil. Eng. 18, 319 (2010).

[16] A. Wongsarnpigoon, W.M. Grill, J. Neural Eng. 7, 046009 (2010).

[17] C.R. Butson, C.C. McIntyre, Clin. Neurophysiol. 118, 1889 (2007).

[18] C.C. McIntyre, W.M. Grill, J. Neurophysiol. 88, 1592 (2002).

[19] R.K. Shepherd, E. Javel, Hear. Res. 130, 171 (1999).

[20] R.P. Carlyon, A. van Wieringen, J.M. Deeks, C.J. Long, J. Lyzenga, J. Wouters, Hear. Res. 205, 210 (2005).

[21] E.B. Montgomery Jr., J.T. Gale, Neurosci. Biobeh. Rev. 32, 388 (2008).

[22] E.B. Montgomery Jr., Parkinsonism Relat. Disord. 13, 455 (2007).

[23] W.M. Grill, A.N. Snyder, S. Miocinovic, Neuroreport 15, 1137 (2004).

[24] G. Foffani, A. Priori, M. Egidi, P. Rampini, F. Tamma, E. Caputo, K.A. Moxon, S. Cerutti, S. Barbieri, Brain 126, 2153 (2003). 
[25] L.S. Borkowski, Phys. Rev. E 80, 051914 (2009).

[26] L.S. Borkowski, Phys. Rev. E 82, 041909 (2010).

[27] L.S. Borkowski, Phys. Rev. E 83, 051901 (2011).

[28] L.S. Borkowski, Acta Phys. Pol. A 122, 776 (2012).

[29] R.P. Morse, E.F. Evans, Nature Med. 2, 928 (1996).

[30] F. Moss, F. Chiou-Tan, R. Klinke, Nature Med. 2, 860 (1996).

[31] D.E. O'Gorman, J.A. White, C.A. Shera, J. Assoc. Res. Otolaryngol. 10, 251 (2009).

[32] D.E. O'Gorman, H.S. Colburn, C.A. Shera, J. Acoust. Soc. Am. 128, 300 (2010).

[33] R. FitzHugh, Biophys. J. 1, 445 (1961).

[34] J.E. Rose, J.R. Brugge, D.J. Anderson, J.E. Hind, J. Neurophysiol. 30, 769 (1967).

[35] E.C. Moxon, Ph.D. Thesis, MIT, 1971.

[36] I.J. Hochmair-Desoyer, E.S. Hochmair, H. Motz, F. Rattay, Neuroscience 13, 553 (1984).

[37] C. van den Honert, P.H. Stypulkowski, Hear. Res. 29, 207 (1987).

[38] L.M. Litvak, B. Delgutte, D.K. Eddington, J. Acoust. Soc. Am. 114, 2079 (2003).

[39] L.M. Litvak, B. Delgutte, D.K. Eddington, J. Acoust. Soc. Am. 114, 2099 (2003).

[40] C.A. Miller, N. Hu, F. Zhang, B.K. Robinson, P.J. Abbas, J. Assoc. Res. Otolaryngol. 9, 122 (2008).

[41] C.M. McKay, K.R. Henshall, Hear. Res. 181, 94 (2003).

[42] P. Prado-Gutierrez, L.M. Fewster, J.M. Heasman, C.M. McKay, R.K. Shepherd, Hear. Res. 215, 47 (2006).

[43] O. Macherey, R.P. Carlyon, A. van Wieringen, J. Wouters, J. Assoc. Res. Otolaryngol. 8, 84 (2007).

[44] J.E. Smit, T. Hanekom, A. van Wieringen, J. Wouters, J.J. Hanekom, Hear. Res. 269, 12 (2010).

[45] A.L. Hodgkin, A.F. Huxley, J. Physiol. (London) 117, 500 (1952).

[46] E.J. Tehovnik, A.S. Tolias, F. Sultan, W.M. Slocum, N.K. Logothetis, J. Neurophysiol. 96, 512 (2006).

[47] N.S. Davidovics, G.Y. Fridman, B. Chiang, C.C.D. Santina, IEEE Trans. Neural Syst. Rehabil. Eng. 19, 84 (2011).
[48] E. Puil, B. Gimbarzevsky, R.M. Miura, J. Neurophysiol. 55, 995 (1986).

[49] B. Hutcheon, Y. Yarom, Trends Neurosci. 23, 216 (2000).

[50] A. van Wieringen, R.P. Carlyon, O. Macherey, J. Wouters, Hear. Res. 220, 49 (2006).

[51] A. van Wieringen, R.P. Carlyon, J. Laneau, J. Wouters, Hear. Res. 200, 73 (2005).

[52] A. van Wieringen, O. Macherey, R.P. Carlyon, J.M. Deeks, J. Wouters, Hear. Res. 242, 154 (2008)

[53] O. Macherey, A. van Wieringen, R.P. Carlyon, J.M. Deeks, J. Wouters, J. Assoc. Res. Otolaryngol. 7, 253 (2006).

[54] B. Agüera y Arcas, A.L. Fairhall, W. Bialek, Neural Comput. 15, 1715 (2003)

[55] A.L. Hodgkin, W.A.H. Rushton, Proc. R. Soc. Lond. B 133, 444 (1946).

[56] D. Noble, R.B. Stein, J. Physiol. 187, 129 (1966).

[57] C. Koch, Biophysics of Computation, Oxford University Press, Oxford 1999, p. 392.

[58] S.Y. Chiu, J.M. Ritchie, R.B. Rogart, D. Stagg, J. Physiol. 292, 149 (1979).

[59] J.D. Sweeney, J.T. Mortimer, D. Durand, IEEE 9th Ann. Conf. Eng. Med. Biol. Soc. 3, 1577 (1987).

[60] A.J. Matsuoka, P.J. Abbas, J.T. Rubinstein, C.A. Miller, Hear. Res. 149, 129 (2000).

[61] R.R. Llinas, U. Ribary, D. Jeanmonod, E. Kronberg, P.P. Mitra, Proc. Natl. Acad. Sci. (USA) 96, 15222 (1999).

[62] W.D. Hutchison, J.O. Dostrovsky, J.R. Walters, R. Courtemanche, T. Boraud, J. Goldberg, P. Brown, J. Neurosci. 24, 9240 (2004).

[63] C.C. McIntyre, P.J. Hahn, Neurobiol. Dis. 38, 329 (2010).

[64] C.R. Butson, C.C. McIntyre, Clin. Neurophysiol. 116, 2490 (2005).

[65] C.A. Bossetti, M.J. Birdno, W.M. Grill, J. Neural Eng. 5, 44 (2008).

[66] B. Tracey, M. Williams, J. Neural Eng. 8, 036017 (2011). 